\title{
Concordância entre ferramentas de triagem nutricional pediátrica em indivíduos com síndrome nefrótica
}

\author{
Concordance between pediatric nutritional screening tools in individuals \\ with nefrotic syndrome
}

\author{
Paula Emília Nunes Ribeiro Bellot ${ }^{\mathbb{D} 1}$, Geovanna Torres de Paiva ${ }^{\mathbb{D} 1}$, Sancha Helena de Lima Vale $\mathbb{D}^{2}$, \\ Richele Janaína de Araújo Machado $\mathbb{D}^{3} \bowtie$ \\ 1 Universidade Federal do Rio Grande do Norte, Hospital Universitário Onofre Lopes, Residência Multiprofissional em Saúde. Natal, Rio Grande do Norte, Brasil. \\ Universidade Federal do Rio Grande do Norte, Departamento de Nutrição. Natal, Rio Grande do Norte, Brasil. \\ ${ }^{3}$ Centro Universitário Christus, Departamento de Nutrição. Fortaleza, Ceará, Brasil.
}

Como citar este artigo (how to cite this article):

Bellot PENR, Paiva GT, Vale SHL, Machado RJA. Concordância entre ferramentas de triagem nutricional pediátrica em indivíduos com síndrome nefrótica (Concordance between pediatric nutritional screening tools in individuals with nefrotic syndrome). Sci Med. 2019;29(4):e33642. https://doi.org/10.15448/1980-6108.2019.4.33642

\section{RESUMO}

OBJETIVO: Avaliar a concordância entre três ferramentas de triagem nutricional validadas para pediatria entre si e a correlação com os parâmetros antropométricos de pacientes hospitalizados com síndrome nefrótica.

MÉTODOS: Estudo transversal com crianças e adolescentes de ambos os sexos internados na enfermaria pediátrica de um hospital universitário, com diagnóstico de síndrome nefrótica. Foram aplicados três instrumentos de triagens nutricionais: triagem de risco para estado nutricional e crescimento (STRONGkids), pontuação pediátrica de desnutrição de Yorkhill (PYMS) e ferramenta de rastreio para a avaliação da desnutrição em pediatria (STAMP). Foram coletados dados de peso, altura e perímetro do braço. A estimativa de concordância relativa entre os instrumentos foi analisada com o teste de Kappa ponderado e as correlações com os parâmetros antropométricos foram avaliadas com o teste de correlação de Spearman.

RESULTADOS: Foram avaliados 11 pacientes, com idade mínima de 2 anos e máxima de 10 anos. As ferramentas de triagem detectaram prevalência de risco nutricional moderado e elevado. Foi observada concordância moderada $(\mathrm{k}=0,47)$ entre a PYMS e a STAMP e não houve correlação entre as triagens e os dados antropométricos. Com relação aos parâmetros antropométricos, $100 \%$ dos participantes tinham peso adequado para idade, $63,6 \%$ dos participantes foram diagnosticados com eutrofia pelo indicador IMC/idade e $81,8 \%$ tinham estatura adequada para idade de acordo com o indicador estatura/idade. Após a análise do perímetro do braço, 27,3\% estavam em risco de desnutrição.

CONCLUSÕES: A despeito de não haver recomendações para uso de triagens nutricionais pediátricas específicas para cada situação clínica, duas das três triagens analisadas neste estudo apresentaram moderada concordância entre elas. Contudo, as triagens testadas não refletiram o estado nutricional antropométrico da população avaliada.

DESCRITORES: Pediatria; síndrome nefrótica; avaliação nutricional.

\section{ABSTRACT}

AIMS: To evaluate the concordance between three validated nutritional screening tools for pediatrics among themselves and the correlation with the anthropometric parameters of patients hospitalized with nephrotic syndrome.

METHOD: Cross-sectional study with children and adolescents of both sexes hospitalized in the pediatric ward of a university hospital and diagnosed with nephrotic syndrome. Three nutritional screening instruments were applied: Screening of Risk for Nutritional Status and Growth (STRONGkids), Paediatric Yorkhill Malnutrition Score (PYMS) and Screening Tool for the Assessment of Malnutrition in Paediatrics (STAMP). Data on weight, height and upper arm perimeter was collected, and three nutritional screening tools were applied: The estimate of relative agreement between the instruments was evaluated with the weighted Kappa test and the correlations with anthropometric parameters were assessed with the Spearman's Correlation Test.

RESULTS: We evaluated 11 patients, with a minimum age of 2 years and a maximum of 10 years. Screening tools detected prevalence of medium and high nutritional risk. Moderate agreement $(\mathrm{k}=0.47)$ was observed between PYMS and STAMP and there was no correlation between the screening tools' result and the anthropometric data. Regarding the anthropometric parameters, $100 \%$ of the participants had adequate weight for age, $63.6 \%$ of the participants were diagnosed with normal weight by the BMI/Age index and $81.8 \%$ had adequate height for age according to the index Height/Age. After analysis of the upper arm perimeter, $27.3 \%$ were at risk of malnutrition.

CONCLUSIONS: Despite the fact that are no recommendations for use of pediatric nutritional screening tools specific to each clinical situation, two of the three screening tools analyzed in this study demonstrated moderate agreement between them. However, the tested tools did not reflect the anthropometric nutritional status of the evaluated population.

KEYWORDS: Pediatrics; nephrotic syndrome; nutrition assessment. 
Abreviaturas: STRONGkids, Triagem de Risco para Estado Nutricional e Crescimento; PYMS, Pontuação Pediátrica de Desnutrição de Yorkhill; STAMP, Ferramenta de Rastreio Para a Avaliação da Desnutrição em Pediatria; IMC, índice de massa corporal.

\section{INTRODUÇÃO}

A síndrome nefrótica é a glomerulopatia mais frequente em crianças e caracteriza-se pelo aumento grave e prolongado da permeabilidade dos glomérulos às proteínas plasmáticas [1]. A incidência dessa doença varia de uma população para outra, pois é influenciada tanto pela geografia como pela etnia, mas está estimada em torno de 2-7 crianças por 100.000 em todo o mundo [2]. Não se tem dados de incidência e prevalência da síndrome nefrótica para a população brasileira, mas sabe-se que está associada a um elevado grau de morbidade [3].

O diagnóstico da síndrome nefrótica é feito quando é identificada a proteinúria maciça $\left(>40 \mathrm{mg} / \mathrm{m}^{2} / \mathrm{h}\right)$, associada à hipoalbuminemia $(<2,5 \mathrm{~g} / \mathrm{dL})$, edema generalizado e hiperlipidemia (colesterol total igual ou acima de $240 \mathrm{mg} / \mathrm{dl}$ ou triglicerídios igual ou acima $200 \mathrm{mg} / \mathrm{dl}$ ) [4]. Este conjunto de sinais e sintomas pode levar ao aparecimento de alterações do metabolismo mineral e ósseo, distúrbios endoteliais e de coagulação. Caso não haja remissão, o risco de apresentar complicações decorrentes da persistência da doença ou da exposição às terapias medicamentosas é maior [5]. Tais complicações envolvem deficiências nutricionais, como depleção de cálcio e potássio e perda proteica na urina, desmineralização óssea, crescimento retardado e hipometabolismo $[1,6]$.

Tendo em vista manter e/ou recuperar o estado nutricional da população pediátrica acometida pela SN, assim como evitar a progressão da lesão renal, o cuidado nutricional é de suma importância. A Associação Brasileira de Nutrição recomenda a sistematização do cuidado nutricional em sete etapas, sendo elas a de triagem nutricional, classificação em níveis de assistência, avaliação nutricional, diagnóstico de nutrição, intervenção, acompanhamento e gestão do cuidado [7]. Existem muitos métodos de avaliação nutricional antropométrica que são usados na prática clínica, no entanto, os que têm o uso mais acessível apresentam limitações, principalmente quando diz respeito ao paciente nefrótico devido às complicações características da doença [8].

Logo, a realização da primeira etapa da sistematização do cuidado nutricional é fundamental, pois a triagem sinaliza precocemente pacientes que estão em risco nutricional e que poderiam beneficiar-se de terapia nutricional. Existem atualmente algumas ferramentas de triagem nutricional pediátrica validadas no mundo, mas a triagem de risco para estado nutricional e crescimento (Screening of Risk for Nutritional Status and Growth - STRONGkids), a pontuação pediátrica de desnutrição de Yorkhill (Paediatric Yorkhill Malnutrition Score - PYMS) e a ferramenta de rastreio para a avaliação da desnutrição em pediatria (Screening Tool for the Assessment of Malnutrition in Paediatrics - STAMP) se destacam pela sua praticidade, aplicabilidade e sensibilidade [9-11]. Apesar de o Ministério da Saúde do Brasil recomendar o uso de ferramentas de triagem nutricional na atenção hospitalar, não existe ainda uma ferramenta de triagem padrão-ouro, sendo comum a comparação entre as já existentes, em busca da identificação de uma ferramenta mais sensível para cada doença $[12,13]$.

Devido às limitações no cuidado do paciente nefrótico, essa população pode beneficiar-se com o uso de ferramenta de triagem para detectar o risco nutricional, para assim prevenir as complicações e melhorar suas condições de saúde e qualidade de vida. Além de diminuir o tempo de internação, o custo e otimizar o cuidado nutricional. Diante disso, a finalidade deste estudo foi avaliar a concordância entre três ferramentas de triagem entre si e suas correlações com os parâmetros antropométricos no contexto da predição de risco nutricional em crianças com síndrome nefrótica.

\section{MÉTODO}

$\mathrm{O}$ estudo realizado teve um delineamento transversal e a coleta de dados ocorreu entre os meses de fevereiro e agosto de 2018 com os pacientes internados na enfermaria pediátrica do hospital universitário Onofre Lopes, localizado em Natal, Rio Grande do Norte.

O processo de amostragem do estudo foi realizado de forma não probabilística sendo elegíveis para participar do estudo todas as crianças e adolescentes internadas no hospital universitário com o diagnóstico de síndrome nefrótica durante o período do estudo. Foram incluídos participantes de ambos os sexos com idade entre 2 e 16 anos e excluídos todos aqueles cujos dados foram coletados após 48 horas da admissão hospitalar, ou que os responsáveis não concordaram com a participação na pesquisa ou os que tinham comorbidades associadas à síndrome nefrótica, como 
diabetes melito tipo 1 e lúpus eritematoso sistêmico, uma vez que a presença dessas doenças poderia ser um fator de viés para as complicações nutricionais. Aqueles que atenderam aos critérios de seleção, tiveram o Termo de Consentimento Livre e Esclarecido e o Termo de Assentimento Livre e Esclarecido assinados. O projeto foi aprovado pelo Comitê de Ética em Pesquisa do Hospital Universitário Onofre Lopes da Universidade Federal do Rio Grande do Norte (parecer no 2.409.882).

Os pacientes foram entrevistados em seus leitos em até 48 horas após a admissão hospitalar. Dados demográficos e dados clínicos da doença como data do diagnóstico, tipo da doença e presença de comorbidades foram coletados em prontuário eletrônico.

As ferramentas de triagem nutricional STAMP, PYMS e STRONGkids foram aplicadas por nutricionista treinada durante a entrevista e a classificação final do risco nutricional foi definida por uma escala de pontos, conforme demonstrado na Tabela 1.

Tabela 1. Classificação de risco nutricional de acordo com a pontuação resultante da aplicação das ferramentas de triagem nutricional.

\begin{tabular}{lccc}
\hline & Risco elevado & Risco moderado & Risco baixo \\
STAMP & $\geq 4$ & $2-3$ & $0-1$ \\
STRONGkids & $4-5$ & $1-3$ & 0 \\
PYMS & $\geq 2$ & 1 & 0 \\
\hline
\end{tabular}

PYMS, pontuação pediátrica de desnutrição de Yorkhill; STAMP, ferramenta de rastreio para a avaliação da desnutrição em pediatria; STRONGkids, triagem de risco para estado nutricional e crescimento.

Os dados antropométricos (peso atual, estatura e perímetro do braço) foram aferidos no mesmo dia da entrevista e o peso habitual foi informado por acompanhante. A aferição do peso atual foi feita em balança digital do tipo plataforma, com capacidade máxima de $200 \mathrm{~kg}$, calibrada, com precisão de 100 $\mathrm{g}$ da marca Soehnle ${ }^{\circledR T M}$. A estatura foi aferida com estadiômetro de parede e o perímetro do braço foi medido com fita métrica inelástica e inextensível. Todas as medidas foram realizadas conforme manual de orientações para a coleta e análise de dados antropométricos do Sistema de Vigilância Alimentar e Nutricional e executadas por nutricionista treinada [14].

A partir da união dos dados demográficos com os dados antropométricos, foram estabelecidos os seguintes indicadores respeitando-se a indicação conforme faixa-etária: Peso/Idade, Peso/Estatura, IMC/Idade e Estatura/Idade. Todos eles foram des- critos em escore $\mathrm{Z}$ e classificados pelas curvas de crescimento da Organização Mundial da Saúde com auxílio dos softwares WHO Anthro versão 3.2.2 e WHO Anthro Plus $[15,16]$. O diagnóstico nutricional antropométrico foi determinado pela interpretação dos escores $\mathrm{Z}$ dos indicadores baseando-se nos pontos de corte estabelecidos pela OMS $(2006 / 2007)[15,16]$ e pela classificação do perímetro do braço preconizado por Frisancho [17].

As variáveis que tiveram distribuição normal de acordo com o teste de Shapiro-Wilk foram descritas em média e desvio padrão e frequências absolutas e relativas. A concordância entre as ferramentas de triagem nutricional foi avaliada com o uso do teste de Kappa Ponderado (k), sendo considerado sem concordância quando o $\mathrm{k}=0$, com baixa concordância $(0,39>\mathrm{k}>0)$, concordância moderada $(0,59>\mathrm{k}>0,39)$, concordância forte $(0,99>\mathrm{k}>0,59)$ e concordância perfeita $(\mathrm{k}=1)$. As correlações estabelecidas entre as triagens nutricionais e os parâmetros antropométricos (IMC/idade e perímetro do braço) foram avaliadas com o teste de correlação de Spearman. Para a análise de concordância entre as ferramentas de triagem nutricional dos dados foi utilizado o programa estatístico R versão 3.5.2 (Viena, Áustria) e para os demais testes estatísticos o programa IBM SPSS Statistics versão 19.0 (Chicago, IL, EUA).

\section{RESULTADOS}

Foram coletados dados de 12 pacientes no total, no entanto 1 participante foi excluído do estudo por ter diagnóstico de Diabete Melito tipo 1. Logo, a amostra foi composta por 11 crianças e adolescentes, com idade entre 2 e 10 anos, sendo a média de 5 anos e 10 meses $( \pm 3,9$ anos). Seis $(63,6 \%)$ pacientes foram do sexo feminino. $\mathrm{O}$ diagnóstico clínico mais presente dentre os pacientes foi o da síndrome nefrótica primária $(90,9 \%)$, causada por lesão renal, e apenas um paciente apresentou diagnóstico da síndrome nefrótica congênita $(9,1 \%)$.

$\mathrm{Na}$ Tabela 2 estão evidenciados os resultados da avaliação do risco nutricional após aplicações das diferentes ferramentas de triagens nutricionais. Observa-se após a análise dos dados que a maior parte dos pacientes foi classificada com risco nutricional moderado quando avaliados através da STAMP $(54,5 \%)$ e a totalidade dos participantes foi classificada com risco moderado pela STRONGkids (100\%). Já a PYMS classificou a maior parte das crianças e adolescentes como estando com risco nutricional elevado $(54,5 \%)$. 
Tabela 2. Classificação de risco nutricional após aplicação das ferramentas de triagens nutricionais.

\begin{tabular}{lccc}
\hline $\begin{array}{l}\text { Classificação de } \\
\text { risco nutricional por } \\
\text { ferramenta }\end{array}$ & $\begin{array}{c}\text { STRONGKids } \\
\mathbf{n}(\%)\end{array}$ & $\begin{array}{c}\text { PYMS } \\
\mathbf{n}(\%)\end{array}$ & $\begin{array}{c}\text { STAMP } \\
\mathbf{n}(\%)\end{array}$ \\
Risco Elevado & 0 & $6(54,5)$ & $5(45,5)$ \\
Risco Moderado & $11(100)$ & $1(9,1)$ & $6(54,5)$ \\
Risco Baixo & 0 & $4(36,4)$ & 0 \\
\hline
\end{tabular}

PYMS, pontuação pediátrica de desnutrição de Yorkhill; STAMP, ferramenta de rastreio para a avaliação da desnutrição em pediatria; STRONGkids, triagem de risco para estado nutricional e crescimento.

Não foi possível realizar a investigação estatística para STRONGkids, em comparação com a STAMP e PYMS, uma vez que o resultado dessa triagem foi o mesmo para todos os participantes. Por outro lado, a PYMS e STAMP apresentaram concordância moderada $(k=0,47)$. Não foi observada correlação entre as ferramentas de triagens nutricionais e os parâmetros antropométricos (indicador IMC/idade e perímetro do braço).

Os pacientes apresentaram média de peso atual de $22,8 \pm 13,1 \mathrm{~kg}$ e peso habitual médio de $20,9 \pm 11,8 \mathrm{~kg}$. Com relação aos indicadores antropométricos, todos os participantes apresentaram peso adequado para a idade; já o indicador peso/estatura identificou $66,7 \%$ dos participantes com eutrofia e 33,3\% com excesso de peso (sobrepeso ou obesidade). A relação da estatura para a idade foi adequada para $9(81,8 \%)$ pacientes, e tida como baixa para $2(18,2 \%)$ dos investigados. $\mathrm{O}$ indicador IMC/idade identificou que $7(63,6 \%)$ pacientes estavam eutróficos, $3(27,3 \%)$ estavam com sobrepeso ou obesidade e $1(9,1 \%)$ com magreza. Por fim, a adequação do perímetro braquial demonstrou risco de desnutrição para $3(27,3 \%)$ pacientes e risco de obesidade para $1(9,1 \%)$.

\section{DISCUSSÃO}

O presente estudo é relevante e provavelmente, inédito, pois os autores não encontraram outro estudo a aplicar três diferentes ferramentas de triagem nutricional e compará-las com os parâmetros antropométricos de crianças com síndrome nefrótica internadas em um hospital de alta complexidade.

No que diz respeito à faixa etária, este estudo identificou que a maioria das crianças está na faixa de 2 a 4 anos, concordando com outros estudos realizados anteriormente $[1,18,19]$. Na prática clínica dos autores pode-se perceber que é muito comum o diagnóstico desta doença ser feito nessa idade, assim como relataram Lage et al. [1] e Peres et al. [20] nos seus estudos.
Com relação ao diagnóstico clínico dos pacientes, identificou-se que a maioria deles tinham diagnóstico de síndrome nefrótica primária. A doença primária é o diagnóstico mais comum, quando comparada aos outros tipos [18]. E o mesmo foi observado por outro estudo que constatou que $33,4 \%$ das crianças foram acometidas por lesões primárias [21].

O peso e estatura são essenciais para avaliação dos indicadores antropométricos recomendados pelo Ministério da Saúde $[15,16]$. Os parâmetros antropométricos de pacientes com síndrome nefrótica nem sempre refletem o seu real risco nutricional ou estado nutricional, uma vez que podem ser influenciados por alterações próprias da fisiopatologia da doença. A pequena diferença entre o peso atual e o peso habitual encontrada nas crianças e adolescentes avaliados neste estudo pode estar relacionada a presença de edema no momento da admissão hospitalar, visto que o edema é a manifestação clínica mais comum em pacientes com síndrome nefrótica $[1,22]$.

Ao analisar as ferramentas de triagem que podem ser utilizadas na primeira etapa do cuidado nutricional de pacientes pediátricos com síndrome nefrótica, constatamos resultados divergentes entre as ferramentas, sobretudo quando comparada a STRONGkids com as demais. Com o uso dessa ferramenta, todos os participantes foram classificados estando em risco nutricional moderado. A triagem pela STRONGkids possui alta sensibilidade (100\%) e baixa especificidade (7,7\%) de acordo com Wonoputri et al. [23], podendo descrever resultados falso-positivos e implicando em classificar como em risco nutricional pacientes que não estão $[15,22]$. Hulst et al. utilizaram a STRONGkids como ferramenta de triagem em seu estudo e encontraram $54 \%$ das crianças em risco nutricional moderado [9].

A partir da triagem nutricional STAMP, também utilizada neste estudo, observou-se que a maior parte dos participantes foi classificada em risco nutricional moderado e elevado. Em outro estudo realizado com a mesma ferramenta para avaliar crianças hospitalizadas que tivessem qualquer diagnóstico clínico, encontraram risco nutricional elevado para $68 \%$ dos participantes do estudo [24] . Bousquet et al. [25] relataram em seu estudo que a STAMP superestimou o número de crianças em risco nutricional, em contrapartida, McCarthy et al. relataram que a STAMP tem uma alta especificidade e valor preditivo negativo o que reduz o risco de identificação excessiva de crianças com risco nutricional elevado [11].

Em contraste com a STRONGkids e a STAMP, com a ferramenta de triagem PYMS, 54,5\% foram 
classificados em risco nutricional elevado, $36,4 \%$ em risco baixo e apenas um participante estava em risco moderado. Gerasimidis et al. referem que a PYMS produz menos resultados falsos-positivos do que a STAMP e que por esse motivo possui maior valor preditivo positivo, refletindo uma maior probabilidade de que o paciente identificado em determinada classificação de risco nutricional pela PYMS de fato esteja assim [10], sendo, portanto, uma ferramenta muito confiável $[24,26]$.

Visto que não existe uma ferramenta de triagem de risco nutricional para a população pediátrica que seja padrão ouro, faz-se necessária a comparação entre as já existentes para que se identifique aquela que mais se adequa à população em questão. No caso dos pacientes com síndrome nefrótica, a STRONGkids não apresentou concordância com as outras ferramentas, enquanto a STAMP e a PYMS apresentaram concordância moderada entre si, corroborando com um estudo com crianças hospitalizadas que utilizou as mesmas ferramentas de triagem nutricional [10]. Apesar de a STRONGkids estar bem estabelecida na prática clínica como uma ferramenta de triagem nutricional de estrutura simples, com praticidade na aplicação e facilidade de uso no ambiente hospitalar, apresenta uma sensibilidade muito alta e especificidade muito baixa, fazendo com que essa ferramenta possivelmente não seja a melhor escolha de uso para a população em questão [26].

Apesar de serem amplamente utilizados na prática clínica, os dados de peso e altura não refletem necessariamente a composição corporal no caso de indivíduos com síndrome nefrótica, por este motivo o presente estudo também avaliou o perímetro do braço e sua adequação segundo Frisancho [17]. O resultado encontrado foi de $63,6 \%$ de adequação do perímetro braquial e risco de desnutrição em $27,3 \%$ dos casos. Esse dado sugere que o perímetro do braço seja um marcador mais sensível para identificar risco de desnutrição nessa população, uma vez que esse parâmetro é menos influenciado pelo balanço hídrico corporal do que os indicadores baseados em peso [27].
No presente estudo nenhuma das ferramentas de triagem nutricional apresentou correlação com os parâmetros antropométricos. Para esta análise, provavelmente seria necessário um maior número de participantes.

A principal limitação deste estudo foi o tamanho da amostra. Este número foi restrito devido ao fato de que o desenho metodológico previa a realização do estudo em um período pré-estabelecido e o número de internações hospitalares por síndrome nefrótica nesse período foi abaixo do que historicamente vinha sendo constatado na unidade hospitalar de escolha. Um viés pode ter ocorrido em razão do peso atual dos participantes ser aferido independente da presença de edema. Isto ocorreu por não encontrarmos na literatura científica fórmulas matemáticas que contabilizem o valor do edema em relação ao peso de crianças e adolescentes, assim como os estudos prévios utilizavam o peso sem edema clinicamente visível $[28,29]$.

Acredita-se que esse estudo reforça a necessidade de se estabelecer como fundamental a realização da triagem nutricional no cuidado de pacientes com síndrome nefrótica possibilitando assim uma melhor assistência nutricional. Além disso, incentiva a realização de mais estudos que possam detectar a ferramenta de triagem mais sensível ou validar instrumentos específicos para essa população.

\section{NOTAS}

Apoio financeiro

Este estudo não recebeu apoio financeiro de fontes externas.

Declaração de conflito de interesses

Os autores declaram não haver conflitos de interesses relevantes ao conteúdo deste estudo.

Contribuições dos autores

Todos os autores fizeram contribuições substanciais para concepção, ou delineamento, ou aquisição, ou análise ou interpretação de dados; e redação do trabalho ou revisão crítica; e aprovação final da versão para publicação.

Disponibilidade dos dados e responsabilidade pelos resultados

Todos os autores declaram ter tido total acesso aos dados obtidos e assumem completa responsabilidade pela integridade destes resultados.

\section{REFERÊNCIAS}

1. Lage FB. Recomendação proteica para crianças com síndrome nefrótica. Rev Bras Nutr Clin. 2014;29(4):360-6.

2. Hussain N, Zello JA, Vasilevska-Ristovska J, Banh TM, Patel VP, Patel P, Battiston CD, Hebert D, Licht CP, Piscione TD, Parekh RS. The rationale and design of Insight into Nephrotic Syndrome: Investigating Genes, Health and Therapeutics (INSIGHT): a prospective cohort study of childhood nephrotic syndrome. BMC Nephrol. 2013;14:25. https://doi:10.1186/1471-2369-14-25 
3. Niaudet P, Boyer O. Idiopathic nephrotic syndrome in children: clinical aspects. In: Avner E, Harmon W, Niaudet P, Yoshikawa N, Emma F, Goldstein S, editors. Pediatric nephrology. Berlin: Springer; 2016. p. 839-82. https://doi. org/10.1007/978-3-662-43596-0_24

4. Phan V, Blydt-Hansen T, Feber J, Alos N, Arora S, Atkinson S, Bell L, Clarson C, Couch R, Cummings EA, Filler G, Grant RM, Grimmer J, Hebert D, Lentle B, Ma J, Matzinger M, Midgley J, Pinsk M, Rodd C, Shenouda N, Stein R, Stephure D, Taback S, Williams K, Rauch F, Siminoski K, Ward LM; Canadian STOPP Consortium. Skeletal findings in the first 12 months following initiation of glucocorticoid therapy for pediatric nephrotic syndrome. Osteoporos Int. 2014;25(2):627-37. https://doi:10.1007/s00198-013-2466-7

5. Agrawal S, Zaritsky JJ, Fornoni A, Smoyer WE. Dyslipidaemia in nephrotic syndrome: mechanisms and treatment. Nat Rev Nephrol. 2018;14(1):57-70. https://doi:10.1038/nrneph.2017.155

6. El-Mashad GM, El-Hawy MA, El-Hefnawy SM, Mohamed SM. Bone mineral density in children with idiopathic nephrotic syndrome. J Pediatr. 2017;93(2):142-7. https://doi:10.1016/j.jped.2016.05.010

7. Fidelix MSP, organizador. Manual Orientativo: sistematização do cuidado de nutrição. São Paulo: Associação Brasileira de Nutrição; 2014.

8. Fortes Almeida A, Lima Gusmao-Sena MH, Gonzaga Oliveira LC, Santana Gomes T, Neves do Nascimento TV, Nunes Gobatto AL, Ramos Sampaio L, Barreto-Medeiros JM. Inflammatory, nutritional and clinical parameters of individuals with chronic kidney disease undergoing conservative treatment. Nutr Hosp. 2015;32(3):1376-81. https://doi:10.3305/nh.2015.32.3.9125

9. Hulst JM, Zwart H, Hop WC, Joosten KF. Dutch national survey to test the STRONGkids nutritional risk screening tool in hospitalized children. Clin Nutr. 2010;29(1):106-11. https://doi:10.1016/j.clnu.2009.07.006

10. Gerasimidis K, Keane O, Macleod I, Flynn DM, Wright CM. A four-stage evaluation of the Paediatric Yorkhill Malnutrition Score in a tertiary paediatric hospital and a district general hospital. Br J Nutr. 2010;104(5):751-6. https://doi:10.1017/S0007114510001121

11. McCarthy H, Dixon M, Crabtree I, Eaton-Evans MJ, McNulty H. The development and evaluation of the Screening Tool for the Assessment of Malnutrition in Paediatrics (STAMP ${ }^{\circ}$ ) for use by healthcare staff. J Hum Nutr Diet. 2012;25(4):311-8. https://doi:10.1111/j.1365-277X.2012.01234.x

12. Raslan M, Gonzalez MC, Dias MCG, Paes-Barbosa FC, Cecconello I, Waitzberg DL. Aplicabilidade dos métodos de triagem nutricional no paciente hospitalizado. Rev Nutr. 2008;21(5): 553-61. https://doi:10.1590/S141552732008000500008

13. Brasil. Ministério da Saúde. Secretaria de Atenção à Saúde. Departamento de Atenção Especializada e Temática. Manual de Terapia Nutricional na Atenção Especializada Hospitalar no âmbito do Sistema Único de Saúde - SUS. Brasília: Ministério da Saúde; 2016. https://doi.org/10.5123/s1679-49742019000100016

14. Brasil. Ministério da Saúde. Secretaria de Atenção à Saúde. Departamento de Atenção Básica. Orientações para a coleta e análise de dados antropométricos em serviços de saúde: norma técnica do sistema de vigilância alimentar e nutricional - SISVAN. Brasília: Ministério da Saúde; 2011. https://doi.org/10.5123/s1679-49742019000200006

15. de Onis M, Onyango AW, Borghi E, Siyam A, Nishida C, Siekmann J. Development of a WHO growth reference for school-aged children and adolescents. Bull World Health Organ. 2007;85(9):0660-7. https://doi:10.2471/BLT.07. 043497

16. WHO Multicentre Growth Reference Study Group. WHO Child Growth Standards based on length/height, weight and age. Acta Paediatr Suppl. 2006;450:76-85. https://doi:10.1080/08035320500495548

17. Frisancho AR. Anthropometric standards for the assessment of growth and nutritional status. Clin Nutrition. 1991;10(2):131-2. https://doi:10.1016/0261-5614(91)90103-J

18. Eddy AA, Symons JM. Nephrotic syndrome in childhood. Lancet. 2003;362(9384):629-39. https://doi:10.1016/S01406736(03)14184-0

19. León DC, Agudelo AM, Ramos J, Ibarra MD. Caracterización clínica del síndrome nefrótico en infantes de Neiva. RFS Revista Facultad de Salud. 2015;7(1):9-16. https://doi:10.25054/rfs.v7i1.174

20. Peres LAB, Bertol MFR. Doenças renais na infância. Rev Med Res. 2012;14(3):186-92.

21. Moreno MPR, García GP. Characteristics of idiopathic nephrotic syndrome at an unusual age in a tertiary-level pediatric hospital in Guadalajara, Jalisco, México. Bol Med Hosp Infant Mex. 2011;68(4):271-7. 
22. Park SJ, Shin J Il. Complications of nephrotic syndrome. Korean J Pediatr. 2011;54(8):322-8. https://doi:10.3345/ kjp.2011.54.8.322

23. Wonoputri N, Djais JTB, Rosalina I. Validity of nutritional screening tools for hospitalized children. J Nutr Metabol. 2014;2014:ID 143649. https://doi:10.1155/2014/143649

24. Ling RE, Hedges V, Sullivan PB. Nutritional risk in hospitalised children: an assessment of two instruments. Clin Nutr ESPEN. 2011;6(3):e153-7. https://doi:10.1016/j.eclnm.2011.01.007

25. Bousquet LA, Stringhini MLF, Mortoza AS. Avaliação nutricional subjetiva global: Instrumentos para triagem em crianças hospitalizadas. Rev Atenção Saúde. 2016;14(47):67-74. https://doi:10.13037/rbcs.vol14n47.3487

26. Aponte Borda AD, Pinzón Espitia OL, Aguilera Otalvaro PA. Tamizaje nutricional en paciente pediátrico hospitalizado: revisión sistemática. Nutrición Hospitalaria. 2018;35(5):1221-8. https://doi:10.20960/nh.1658

27. Mramba L, Ngari M, Mwangome M, Muchai L, Bauni E, Walker AS, Gibb DM, Fegan G, Berkley JA. A growth reference for mid upper arm circumference for age among school age children and adolescents, and validation for mortality: growth curve construction and longitudinal cohort study. BMJ. 2017;358(8117):j3423. https://doi:10.1136/ bmj.j3423

28. Solarin AU, Adekunle MO, Olutekunbi OA, Lamina OM, Aremu OE, Animasahun AB, Njokanma FO. Nutritional Assessment of Children with Nephrotic Syndrome Attending a Tertiary Health Facility: a case control study. Tropical Journal of Nephrology. 2018;13(2):97-104.

29. Oliveira IC, Belangero VMS. Crescimento e composição corporal em crianças e adolescentes com síndrome nefrótica córtico-dependente. J Bras Nefrol. 2009;31(4):252-57. https://doi:10.1590/S0101-28002009000400003 